\title{
Monotonicity and nash implementation in matching markets with contracts
}

Citation for published version (APA):

Haake, C. J., \& Klaus, B. E. (2007). Monotonicity and nash implementation in matching markets with contracts. METEOR, Maastricht University School of Business and Economics. METEOR Research Memorandum No. 058 https://doi.org/10.26481/umamet.2007058

Document status and date:

Published: 01/01/2007

DOI:

10.26481/umamet.2007058

Document Version:

Publisher's PDF, also known as Version of record

\section{Please check the document version of this publication:}

- A submitted manuscript is the version of the article upon submission and before peer-review. There can be important differences between the submitted version and the official published version of record.

People interested in the research are advised to contact the author for the final version of the publication, or visit the DOI to the publisher's website.

- The final author version and the galley proof are versions of the publication after peer review.

- The final published version features the final layout of the paper including the volume, issue and page numbers.

Link to publication

\footnotetext{
General rights rights.

- You may freely distribute the URL identifying the publication in the public portal. please follow below link for the End User Agreement:

www.umlib.nl/taverne-license

Take down policy

If you believe that this document breaches copyright please contact us at:

repository@maastrichtuniversity.nl

providing details and we will investigate your claim.
}

Copyright and moral rights for the publications made accessible in the public portal are retained by the authors and/or other copyright owners and it is a condition of accessing publications that users recognise and abide by the legal requirements associated with these

- Users may download and print one copy of any publication from the public portal for the purpose of private study or research.

- You may not further distribute the material or use it for any profit-making activity or commercial gain

If the publication is distributed under the terms of Article $25 \mathrm{fa}$ of the Dutch Copyright Act, indicated by the "Taverne" license above, 


\title{
Monotonicity and Nash Implementation in Matching Markets with Contracts*
}

\author{
Claus-Jochen Haake $\quad$ Bettina Klaus ${ }^{\ddagger}$
}

April 2007

\begin{abstract}
We consider general two-sided matching markets, so-called matching with contracts markets as introduced by Hatfield and Milgrom (2005), and analyze (Maskin) monotonic and Nash implementable solutions. We show that for matching with contracts markets the stable correspondence is monotonic and implementable. Furthermore, any solution that is Pareto efficient, individually rational, and monotonic is a supersolution of the stable correspondence. In other words, the stable correspondence is the minimal solution that is Pareto efficient, individually rational, and implementable.
\end{abstract}

JEL classification: C62, C78, D78, J41.

Keywords: Matching with Contracts, Monotonicity, Nash implementation, Stability.

\section{Introduction}

We consider a general class of two-sided (many-to-one) matching markets, so-called matching with contracts markets (Hatfield and Milgrom, 2005). ${ }^{1}$ The matching with contracts model contains classical marriage markets (e.g., Gale and Shapley, 1962), college admissions markets (e.g., Roth, 1985), job matching markets (e.g., Kelso and Crawford, 1982), ${ }^{2}$ and certain auction markets (e.g., Milgrom, 2004). Throughout the article, without loss of generality, we model a matching market with contracts as a medical job market consisting of two finite and disjoint sets of agents, which we refer to as doctors and hospitals. Furthermore, there is a set of bilateral contracts between doctors and hospitals that specify the employment conditions (e.g., salary, job profile, retirement plan, etc.). The agents' strict preferences over (feasible, legal, etc.) sets of contracts or allocations completes the description of a matching market with contracts.

In many centralized labor markets, clearinghouses are most often successful if they produce stable allocations (e.g., Roth, 1984a, 1991; Roth and Xing, 1994). Loosely speaking, an allocation is "stable" if it is individually rational [each doctor (hospital) finds

\footnotetext{
${ }^{*}$ B. Klaus thanks the Netherlands Organisation for Scientific Research (NWO) for its support under grant VIDI-452-06-013.

${ }^{\dagger}$ Institute of Mathematical Economics, Bielefeld University, P.O. Box 100131, 33501 Bielefeld, Germany; e-mail: CHaake@wiwi.uni-bielefeld.de

${ }^{\ddagger}$ Corresponding author: Department of Economics, Maastricht University, P.O. Box 616, 6200 MD Maastricht, The Netherlands e-mail: B.Klaus@algec.unimaas.nl

${ }^{1}$ Roth (1984b) considers a more general many-to-many job-matching model with contracts.

${ }^{2}$ Roth and Sotomayor (1990) give a comprehensive and complete survey of these and related two-sided matching models up to 1990 .
} 
the respective contract(s) acceptable] and satisfies no blocking [no hospital can block the allocation by offering an alternative set of contracts that itself and all doctors involved in the new contracts prefer]. It is well-known that for matching markets with sufficient substitutability instabilities can be ruled out: for one-to-one and many-to-one matching markets without money see Gale and Shapley (1962) and Roth (1985), for many-to-one matching markets with money see Kelso and Crawford (1982), and for many-to-many schedule matching see Alkan and Gale (2003). For matching markets with contracts Hatfield and Milgrom (2005) prove that substitutability of hospitals' preferences is sufficient to guarantee stability. In other words, if hospitals' preferences are substitutable, then the stable correspondence that assigns to each matching market with contracts its set of stable matchings is well-defined. ${ }^{3}$

In order to solve a matching problem and determine a stable allocation a centralized clearinghouse would need to know all agents' preferences. An appealing strategic property for solutions that requires that no agent can ever benefit from misrepresenting his/her preferences is strategy-proofness, i.e., truth-telling is a weakly dominant strategy for all agents. Unfortunately, there exists no strategy-proof (single-valued) solution that always assigns stable allocations (Roth, 1982). Phrased differently, stable allocations cannot be reached through weakly dominant "truth-telling equilibria," i.e., truth-telling is not always a weakly dominant strategy for all agents in the direct revelation game. The next question that naturally arises is whether there is another way to obtain stable allocations through strategic interaction, a question that is at the heart of the theory of implementation (for recent surveys see Jackson, 2001; Maskin and Sjöström, 2002). A mechanism consists of a collection of strategy spaces (one for each agent) and an outcome function, which assigns to each profile of chosen strategies a feasible allocation. Together with agents' preferences, which are used to evaluate such allocations, we obtain a game in strategic form. Then, to Nash implement the stable correspondence means that the set of allocations induced by all Nash equilibria coincides with the set of stable allocations. Note that "payoffs" in the above games are reached in two steps: first the outcome function determines an allocation and then each agent evaluates the allocation using individual preferences. Thus, the mechanism can be set up independent of agents' (true) preferences and therefore a centralized clearinghouse would not have to have any particular information about the involved agents and their preferences. In his seminal paper Maskin $(1977,1999)$ introduced a necessary, but not sufficient, condition for Nash implementability: (Maskin) monotonicity. For economies with at least three agents, Moore and Repullo (1990) formulated a necessary and sufficient condition for Nash implementability: monotonicity in combination with a weak no veto power requirement (see Maskin, 1999).

For two-sided marriage and college admissions markets, Kara and Sönmez $(1996,1997)$ show that the stable correspondence is Nash implementable. Sönmez (1996) obtains a corresponding implementability result for so-called generalized matching markets; a class of one-sided matching problems that include marriage and roommate markets (Gale and

\footnotetext{
${ }^{3}$ For many-to-one matching markets with substitutable preferences (e.g., matching with contract markets à la Hatfield and Milgrom, 2005, or the subclass of many-to-one matching markets included in the set of many-to-many job-matching markets considered by Roth, 1984b) the set of stable matchings equals the core. For many-to-many matching markets with substitutable preferences the core may be empty and the relation between the core, the set of stable matchings, and the set of pairwise stable matchings is more complex (see for instance Echenique and Oviedo, 2006). Roth's (1984b) results only apply to pairwise stable matchings.
} 
Shapley, 1962) as well as housing markets (Shapley and Scarf, 1974). In a recent paper Ehlers (2004) obtains positive implementation results in generalized matching markets when agents are allowed to have weak preferences. Apart from the Nash implementability of the stable correspondence, all mentioned articles discuss stability in relation to monotonicity, Pareto efficiency, and individual rationality.

In the present article we focus on Nash implementability of the stable correspondence in matching markets with contracts. We show that the stable correspondence is monotonic (Theorem 1). Moreover, any Pareto efficient, individual rational and monotonic solution is a supersolution of the stable correspondence (Theorem 2). The latter result implies that the stable correspondence is the smallest possible monotonic solution (Corollary 1), which implies that by Maskin's fundamental result it is impossible to implement any subcorrespondence (e.g., single-valued) of the stable correspondence. Finally, by verifying Moore and Repullo's (1990) "condition $\mu$ " we prove that the stable correspondence is Nash implementable in matching markets with contracts with more than two agents (Theorem 3). Theorem 4 complements Theorem 3 by showing that the implementation of stable two agent contracts (the two agent case) is not possible. Finally, apart from obtaining various monotonicity and implementation results concerning the stable correspondence in matching with contracts markets, we also provide an alternative proof technique for the Nash implementability result. To be more precise, all previous papers that demonstrate implementability of the stable correspondence in various matching models (Ehlers, 2004; Kara and Sönmez, 1996, 1997; Sönmez, 1996) use Yamato's (1992) "essential monotonicity" of the stable correspondence. We apply Moore and Repullo's (1990) implementability condition and show that alternatively monotonicity and a particular no veto power property of the stable correspondence assures its implementability (see Section 4 for further details). In addition, we prove that Kara and Sönmez's (1997) results for many-to-one matching markets with responsive preferences still hold on the larger domain of substitutable preferences (a domain of which it is known that various results concerning stable matchings that hold for many-to-one matching markets with responsive preferences do not hold; see for instance Martínez et al., 1991).

\section{Matching Markets with Contracts and Stability}

\subsection{Doctors, Hospitals, and Contracts}

We consider a model, in which doctors are matched to hospitals. Let $D$ denote the finite set of doctors, $H$ the finite set of hospitals, and $N=D \cup H$ the set of all agents. By $d$ we denote a generic doctor, by $h$ a generic hospital, and by $i, j$ generic agents.

To model the typical feature of a job-matching market, we assume that each doctor can be matched to at most one hospital, whereas each hospital may be matched to several doctors. A (bilateral) contract specifies a match between one doctor and one hospital and further terms of employment such as, for instance, salary, working time and schedule, social benefits, or a combination of these and additional contract terms. Formally, the set of contracts is described by a finite set $X$ together with a mapping $\mu=\left(\mu_{D}, \mu_{H}\right): X \longrightarrow$ $D \times H$ that specifies the bilateral structure of each contract. So, for any contract $x \in X$, $\mu(x)=(d, h)$ means that contract $x$ is established between doctor $d$ and hospital $h$. Note that for two contracts $x, x^{\prime} \in X, x \neq x^{\prime}$, with $\mu(x)=\mu\left(x^{\prime}\right), x$ and $x^{\prime}$ specify different terms of employment for the same doctor in the same hospital. 
If all hospitals offer the same set of employment specifications $K$ to all doctors, then the set of contracts $X$ can be represented as a Cartesian product $X=D \times H \times K$ with $\mu$ being the projection on $D \times H$. An example of such an employment specification $K$ would be a salary scale that by law has to be employed by all hospitals. However, note that hospitals may not necessarily use the same employment specification: a public hospital may not be able to offer the same salaries and social benefits as a private clinic, but on the other hand it may offer employment in fields of specialization that cannot be offered by a private clinic. Furthermore, not all hospitals need to offer the same employment specifications to all doctors, for instance because hospitals condition their employment specifications on the doctors' qualifications. Also, we do not assume that $\mu$ is surjective. Thus, a doctor $d$ might not have any contract with some hospital $h$, for instance, because his field of specialization does not qualify him for any position offered by $h$. For each doctor it is always possible to reject any contract, that is to stay unemployed. We denote such a null contract by $\emptyset$. For each hospital it is always possible to reject any set of contracts, that is to keep positions vacant. We refer to the specific situation in which a hospital does not employ any doctors as a null contract, denoted by $\emptyset$.

\subsection{Doctors' and Hospitals' Preferences}

For $d \in D$ let $X_{d}:=\mu_{D}^{-1}(d)$ be the set of contracts in which doctor $d$ is matched to some hospital, i.e., which are feasible for doctor $d$. Each doctor $d \in D$ has a preference relation $R_{d}$ over feasible contracts, which is a total order over $X_{d} \cup\{\emptyset\} .{ }^{4}$ Alternatively, we interpret doctor $d$ 's preferences over $X_{d} \cup\{\emptyset\}$ as preferences over doctor $d$ 's sets of feasible contracts $\mathcal{X}_{d}:=\left\{\{x\} \mid x \in X_{d} \cup\{\emptyset\}\right\}$.

Recall that $\emptyset$ can either describe the null contract or the empty set. Hence, the feasible contract set $\mathcal{X}_{d}=\{\emptyset\}$ contains one feasible contract while the feasible contract set $\mathcal{X}_{d}=\emptyset$ is empty. In the sequel, it will simplify notation if we allow for the notation " $\emptyset$ " even though we mean " $\{\emptyset\}$ " - since the null contract is always available to any agent no confusion should arise.

For $h \in H$ let $X_{h}:=\mu_{H}^{-1}(h)$ be the set of contracts in which hospital $h$ is matched to some doctor. No hospital $h$ is allowed to have more than one contract with a doctor at the same time. Therefore, we define the sets of feasible contracts for hospital $h$ by $\mathcal{X}_{h}:=$ $\left\{X^{\prime} \subseteq X_{h} \mid\right.$ for all $\left.d \in D,\left|X^{\prime} \cap X_{d}\right| \leq 1\right\}$. Note that $X^{\prime} \in \mathcal{X}_{h}$ implies for all $Y^{\prime} \subseteq X^{\prime}$, $Y^{\prime} \in \mathcal{X}_{h}$. Each hospital $h \in H$ has a preference relation $R_{h}$ over sets of feasible contracts, which is a total order over $\mathcal{X}_{h}$.

To summarize, any agent $i$ 's preference relation can be represented by a total order over $\mathcal{X}_{i}$. We denote the set of all possible total orders for agent $i$ by $\mathcal{R}_{i}$. Since preference relation $R_{i} \in \mathcal{R}_{i}$ is a total order, it induces a well-defined choice correspondence $C_{i}$ : $2^{X} \Longrightarrow X_{i} \cup\{\emptyset\}$ that assigns to each set of contracts $X^{\prime} \subseteq X$ agent $i$ 's most preferred feasible set of contracts available for it in $X^{\prime} \cup\{\emptyset\}$, i.e., $C_{i}\left(X^{\prime}\right) \subseteq X_{i}, C_{i}\left(X^{\prime}\right) \in \mathcal{X}_{i}$, and for all $Y \subseteq X_{i}, Y \in \mathcal{X}_{i}, C_{i}\left(X^{\prime}\right) R_{i} Y$. Since $C_{d}\left(X^{\prime}\right)$ is single-valued, we alternatively identify the set $C_{d}\left(X^{\prime}\right)$ with its unique element. We write $C_{D}\left(X^{\prime}\right):=\bigcup_{d \in D} C_{d}\left(X^{\prime}\right)$ and $C_{H}\left(X^{\prime}\right):=\bigcup_{h \in H} C_{h}\left(X^{\prime}\right)$.

\footnotetext{
${ }^{4} \mathrm{~A}$ total order over a set $M$ is a binary relation $R$ that satisfies antisymmetry (for all $a, b \in M$, if $a R b$ and $b R a$, then $a=b$ ), transitivity (for all $a, b, c \in M$, if $a R b$ and $b R c$, then $a R c$ ), and comparability (for all $a, b \in M, a R b$ or $b R a$ ). By $P$ we denote the asymmetric part of $R$. Hence, given $a, b \in M, a P b$ means that $a$ is strictly preferred to $b$; $a R b$ means that $a P b$ or $a=b$ and that $a$ is weakly preferred to $b$.
} 
In Appendix A, we illustrate how various well-known two-sided matching markets can be modeled as matching with contracts markets.

\subsection{Matching Markets with Contracts, Allocations, and Solutions}

Since the set of contracts $X$ and the set of agents $N$ remain fixed throughout this study, we can denote a matching market (with contracts) by a preference profile $R=\left(R_{i}\right)_{i \in N} \in$ $\prod_{i \in N} \mathcal{R}_{i}$. We denote the set of all preference profiles by $\mathcal{R}=\prod_{i \in N} \mathcal{R}_{i}$. If not otherwise specified, we denote the associated profile of choice correspondences for $R \in \mathcal{R}$ by $\left(C_{i}\right)_{i \in N}$.

For any subset of contracts $Y \subseteq X$, let $Y_{i}:=\left(Y \cap X_{i}\right)$ denote the set of real contracts available for agent $i$ in $Y$. If $Y_{i}=\emptyset$, then only the null contract is available. An allocation is a set $A \subseteq X$ of contracts such that for all agents all sets of contracts available in $A$ are feasible, i.e., for all agents $i \in N, A_{i} \in \mathcal{X}_{i}$. Since contracts are bilateral, $A=\bigcup_{d \in D} A_{d}=$ $\bigcup_{h \in H} A_{h}$. We denote the set of allocations by $\mathcal{A}$. Clearly, all preference relations $R_{i}$ induce weak preferences over allocations in a natural way. We use the same notation for preferences over feasible contracts/contract sets and allocations: for all agents $i \in N$ and allocations $A, A^{\prime} \in \mathcal{A}, A R_{i} A^{\prime}$ if and only if $A_{i} R_{i} A_{i}^{\prime}$.

A solution $\varphi$ is a correspondence $\varphi: \mathcal{R} \Longrightarrow \mathcal{A}$ that assigns to each matching market $R$ a set of allocations $\varphi(R)$. Next we discuss two basic properties for solutions: Pareto efficiency and individual rationality.

An allocation $A \in \mathcal{A}$ is Pareto efficient for matching market $R \in \mathcal{R}$ if there is no other allocation $A^{\prime} \in \mathcal{A}$ such that for all $i \in N, A^{\prime} R_{i} A$ and for some $j \in N, A^{\prime} P_{j} A$. A solution $\varphi$ is Pareto efficient if it only assigns sets of Pareto efficient allocations.

Since all contracts are based on voluntary participation, at any allocation $A \in \mathcal{A}$ each agent $i$ who is assigned a set of real contracts $A_{i} \neq \emptyset$ can reject some or all contracts in $A_{i}$. Thus, an allocation $A \in \mathcal{A}$ is individually rational for matching market $R \in \mathcal{R}$ if for all $i \in N, C_{i}(A)=A_{i}$ or alternatively, $C_{D}(A)=C_{H}(A)=A$. A solution $\varphi$ is individually rational if it only assigns sets of individually rational allocations.

\subsection{Stability: Stable Matchings and Substitutable Preferences}

As described in the Introduction, an important criterion for an allocation to be accepted as final outcome in a job-matching market is stability. Consider a matching market $R \in \mathcal{R}$.

First, since the matching markets we consider here are based on voluntary participation, a necessary condition for allocation $A$ to be stable is individual rationality:

(IR) $C_{D}(A)=C_{H}(A)=A$.

Second, given that allocation $A$ is individually rational, we assume that no hospital can block allocation $A$ together with a set of doctors, i.e., there is no alternative set of contracts $X^{\prime} \in \mathcal{X}_{h}$ such that $X^{\prime} P_{h} A_{h}$ and for all doctors $d \in D$ that are being offered a new contract $x_{d}^{\prime}$ with hospital $h, x_{d}^{\prime} P_{d} A_{d}$. Using the associated profile of choice correspondences, "no blocking" of hospitals can be expressed as: there is no hospital $h$ and no set of contracts $X^{\prime} \in \mathcal{X}_{h}, X^{\prime} \neq A_{h}$, such that $C_{h}\left(A \cup X^{\prime}\right)=X^{\prime}$ and for all doctors $d \in D$ that are being offered a new contract $x_{d}^{\prime}$ with hospital $h, C_{d}\left(A \cup X^{\prime}\right)=x_{d}^{\prime}$. Note that for all doctors $d \in D$ that are being offered the same contract or that are not being offered a contract in $X^{\prime}$, by individual rationality, $C_{d}\left(A \cup X^{\prime}\right)=A_{d}$. Thus, $\bigcup_{d \in D} C_{d}\left(A \cup X^{\prime}\right) \supseteq X^{\prime}$. Hence, we can formulate no blocking by hospitals comprehensively as follows. 
(NB) There is no hospital $h$ and set of contracts $X^{\prime} \in \mathcal{X}_{h}, X^{\prime} \neq C_{h}(A)$, such that $X^{\prime}=C_{h}\left(A \cup X^{\prime}\right) \subseteq C_{D}\left(A \cup X^{\prime}\right)$.

An allocation $A \in \mathcal{A}$ is stable if it is individually rational (IR) and no hospital can block allocation $A$ together with a set of doctors (NB). By $S(R) \subseteq \mathcal{A}$ we denote the set of stable allocations for matching market $R$. It is a standard observation in (many-to-one) matching theory that stable allocations are at the same time core allocations in the sense that no coalition of hospitals and doctors can find another allocation that contains contracts between members of the coalition that they all weakly and some strictly prefer. Thus, similarly as in other matching models, the core equals the set of stable matchings. The stable correspondence $S: \mathcal{R} \Longrightarrow \mathcal{A}$ assigns to each matching market $R$ the set of stable matchings $S(R)$. Note that for the general domain of matching markets specified so far stability is not guaranteed, i.e., there exist matching markets $R \in \mathcal{R}$ with $S(R)=\emptyset$ (see Hatfield and Milgrom, 2005, Theorem 5). Hatfield and Milgrom (2005) prove that the set of stable matchings is non-empty if all hospitals' preferences are substitutable; loosely speaking, a hospital has substitutable preferences if it does not consider complementarities in the sets of contracts it can offer. To be precise, the substitutable preference condition states that if a contract is chosen by a hospital from some set of available contracts, then that contract is still chosen by the hospital from a smaller set of available contracts that include it. Formally, hospital $h$ 's preferences $R_{h}$ are substitutable if

(SUB) for the associated choice correspondence $C_{h}$ and all sets of contracts

$$
X^{\prime} \subseteq Y^{\prime} \in \mathcal{X}_{h}, X^{\prime} \cap C_{h}\left(Y^{\prime}\right) \subseteq C_{h}\left(X^{\prime}\right) .
$$

Equivalently one can formulate substitutability as follows (see Hatfield and Milgrom, 2005). If a contract is not chosen by a hospital from some set of available contracts, then that contract is still not chosen by the hospital from a larger set of available contracts. For any set of contracts $X^{\prime} \in \mathcal{X}_{h}, N C_{h}\left(X^{\prime}\right):=X^{\prime} \backslash C_{h}\left(X^{\prime}\right)$ denotes the set of all contracts that are not chosen from set $X^{\prime}$ by choice correspondence $C_{h}$. One can easily prove that condition (SUB) is equivalent to the following condition (SUB').

(SUB') For all sets of contracts $X^{\prime} \subseteq Y^{\prime} \in \mathcal{X}_{h}, N C_{h}\left(X^{\prime}\right) \subseteq N C_{h}\left(Y^{\prime}\right)$.

Hatfield and Milgrom (2005) showed that the set of stable matchings is non-empty if hospitals' preferences are substitutable. Since for our later results the non-emptiness of the stable correspondence is important and substitutability is a reasonable assumption for many matching markets, from now on the domain of substitutable preferences is our reference domain.

\section{Monotonicity and Nash Implementation}

So far we have described all ingredients for the implementation environment, which is given by the set of agents $N$, the set of alternatives (allocations) $\mathcal{A}$, and the set of preference profiles $\mathcal{R}$. Before focusing on the Nash implementability of the stable correspondence, we consider a necessary condition for Nash implementability (Maskin, 1999) that in itself has a normative appeal: (Maskin) monotonicity. 


\subsection{Monotonicity}

Before introducing monotonicity, we need some standard terms and notation. For any agent $i \in N$, preference relation $R_{i} \in \mathcal{R}_{i}$, and allocation $A \in \mathcal{A}$, the lower contour set of $R_{i}$ at $A$ is $L_{i}\left(R_{i}, A\right):=\left\{A^{\prime} \in \mathcal{A} \mid A R_{i} A^{\prime}\right\}$.

Next, we define monotonic transformations. Loosely speaking, for any preference profile $R$ and any allocation $A$, if at a preference profile $R^{\prime}$ all agents $i \in N$ consider their allotment $A_{i}$ to be (weakly) better, then $R^{\prime}$ is a monotonic transformation of $R$ at $A$. Formally, for preference profiles $R, R^{\prime} \in \mathcal{R}$ and allocation $A \in \mathcal{A}, R^{\prime}$ is a monotonic transformation of $R$ at $A$ if for all $i \in N, L_{i}\left(R_{i}, A\right) \subseteq L_{i}\left(R_{i}^{\prime}, A\right)$. By $M T(R, A)$ we denote the set of all monotonic transformations of $R$ at $A$. For agent $i \in N$, preference relation $R_{i}^{\prime} \in \mathcal{R}_{i}$, and preference profile $R \in \mathcal{R}$, we obtain preference profile $\left(R_{i}^{\prime}, R_{-i}\right)$ by replacing $R_{i}$ at $R$ by $R_{i}^{\prime}$. By $M_{i}(R, A)$ we denote the set of all unilateral monotonic transformations of $R$ at $A$, i.e., monotonic transformations of $R$ at $A$ of the specific form $\left(R_{i}^{\prime}, R_{-i}\right)$.

A solution $\varphi$ is monotonic if an allocation $A$ that is chosen at preference profile $R$ is also chosen at a preference profile $R^{\prime}$ where $A$ is considered (weakly) better by all agents. Formally, a solution $\varphi$ is monotonic if for all preference profiles $R, R^{\prime} \in \mathcal{R}, A \in \varphi(R)$ and $R^{\prime} \in M T(R, A)$ imply $A \in \varphi\left(R^{\prime}\right)$. As discussed in the next subsection, monotonicity is one of the key concepts in implementation theory. Here, we first focus on the implication that monotonicity has on solutions.

Assume that for a matching market $R \in \mathcal{R}$, a given allocation $A \in \mathcal{A}$ and some set of contracts $X^{\prime} \subseteq X$, some agent $j \in N$ considers the contract or the set of contracts $A_{j}$ to be optimal in $A \cup X^{\prime}$, i.e., $C_{j}\left(A \cup X^{\prime}\right)=A_{j}$. Then, $A_{j}$ becoming even better according to agent $j$ 's preferences implies that $A_{j}$ is still optimal for $j$. In other words, if $A_{j}$ is chosen from $A \cup X^{\prime}$ by agent $j$ at the original preferences $R_{j}$, then $A_{j}$ is still chosen by $j$ after a monotonic transformation of $R_{j}$. We summarize this useful fact in the following lemma.

Lemma 1. Let $R \in \mathcal{R}, j \in N, A \in \mathcal{A}$, and $X^{\prime} \subseteq X$ be such that $C_{j}\left(A \cup X^{\prime}\right)=A_{j}$. Then for $R^{\prime} \in M T_{j}(R, A)$ and agent $j$ 's associated choice correspondence $C_{j}^{\prime}, C_{j}^{\prime}\left(A \cup X^{\prime}\right)=A_{j}$.

Theorem 1. The stable correspondence $S$ is monotonic.

Proof: Consider a matching market $R \in \mathcal{R}$ and a stable set of contracts $A \in S(R)$. Let $R^{\prime}$ be a monotonic transformation of $R$ at $A$, i.e., $R^{\prime} \in M T(R, A)$. In order to show that the stable correspondence is monotonic, we need to show that $A \in S\left(R^{\prime}\right)$.

Denote the associated profile of choice correspondences for $R^{\prime}$ by $\left(C_{i}^{\prime}\right)_{i \in N}$.

Unilateral monotonic transformations: First, assume that for some $j \in N, R^{\prime} \in$ $M T_{j}(R, A)$. Hence, for all $i \in N \backslash\{j\}, C_{i}^{\prime}=C_{i}$.

Part (IR): First, we show that allocation $A$ is individually rational at matching market $R^{\prime}$. Since $A \in S(R)$, by (IR) $C_{j}(A)=A_{j}$. Thus, by Lemma $1, C_{j}^{\prime}(A)=A_{j}$, and therefore, $C_{j}^{\prime}(A)=C_{j}(A)$. Recall that for all $i \in N, C_{i}^{\prime}(A)=C_{i}(A)$. Hence, $A$ being individually rational at $R\left[(\mathrm{IR}) C_{D}(A)=C_{H}(A)=A\right]$ implies that $A$ is individually rational at $R^{\prime}$ $\left[(\mathrm{IR}) C_{D}^{\prime}(A)=C_{H}^{\prime}(A)=A\right]$.

Part (NB): Next, we prove that (NB) holds for allocation $A$ at matching market $R^{\prime}$. Suppose, by contradiction, that (NB) does not hold after the monotonic transformation, i.e., there exists a hospital $h \in H$ and a set of contracts $X^{\prime} \in \mathcal{X}_{h}, X^{\prime} \neq C_{h}^{\prime}(A)$, such that $X^{\prime}=C_{h}^{\prime}\left(A \cup X^{\prime}\right) \subseteq C_{D}^{\prime}\left(A \cup X^{\prime}\right)$. 
If $j \in D$, then (NB) for matching market $R$ implies $X^{\prime}=C_{h}\left(A \cup X^{\prime}\right) \not \subset C_{D}\left(A \cup X^{\prime}\right)$. Hence, since $A \in \mathcal{A}$ and $X^{\prime} \in \mathcal{X}_{h}$, agent $j$ can at $A \cup X^{\prime}$ choose contract $A_{j}$ or the unique contract $x_{j}^{\prime} \in X^{\prime} \cap X_{j}, x_{j}^{\prime} \neq A_{j}$. Since for all $d \in D \backslash\{j\}, C_{d}=C_{d}^{\prime}, X^{\prime} \subseteq C_{D}^{\prime}\left(A \cup X^{\prime}\right)$ and $X^{\prime} \nsubseteq C_{D}\left(A \cup X^{\prime}\right)$ imply $C_{j}^{\prime}\left(A \cup X^{\prime}\right)=x_{j}$ and $C_{j}\left(A \cup X^{\prime}\right)=A_{j}$. This contradicts Lemma 1.

Since condition (NB) does not involve any agent in $H \backslash\{h\}$, it is clear that if $j \in H$, then $j=h$. Now, $(\mathrm{NB})$ for matching market $R$ implies $X^{\prime} \neq C_{j}\left(A \cup X^{\prime}\right)$. If $C_{j}\left(A \cup X^{\prime}\right)=A_{j}$, then $C_{j}^{\prime}\left(A \cup X^{\prime}\right)=X^{\prime}$ contradicts Lemma 1. Hence, $C_{j}\left(A \cup X^{\prime}\right)=Y^{\prime} \in \mathcal{X}_{j} \backslash\left\{A_{j}, X^{\prime}\right\}$. Since $Y^{\prime} \subseteq A \cup X^{\prime}, C_{j}\left(A \cup Y^{\prime}\right)=Y^{\prime}$. Recall that our assumption that (NB) does not hold for $R^{\prime}$ implies $X^{\prime}\left(=C_{h}^{\prime}\left(A \cup X^{\prime}\right)\right) \subseteq C_{D}^{\prime}\left(A \cup X^{\prime}\right)$. This means that each doctor $d \in D$ having to choose between two distinct contracts $A_{d} \in A$ and $x_{d}^{\prime} \in X^{\prime}$ always chooses $x_{d}^{\prime}$. Since $Y^{\prime} \subseteq A \cup X^{\prime}$, each doctor $d \in D$ having to choose between two distinct contracts $A_{d} \in A$ and $y_{d}^{\prime} \in Y^{\prime}$ (hence $\left.y_{d}^{\prime} \in X^{\prime}\right)$ always chooses $y_{d}^{\prime}$. Thus, $Y^{\prime} \subseteq C_{D}^{\prime}\left(A \cup Y^{\prime}\right)$. To summarize, there exists a hospital $j$ and a set of contracts $Y^{\prime} \in \mathcal{X}_{h}, Y^{\prime} \neq C_{h}(A)$, such that $Y^{\prime}=C_{h}\left(A \cup Y^{\prime}\right) \subseteq C_{D}\left(A \cup Y^{\prime}\right)$. This contradicts the stability, namely condition (NB), of allocation $A$ in matching market $R$.

General monotonic transformations: So far we have shown that for all unilateral monotonic transformations $R^{\prime} \in M T_{j}(R, A), A \in S\left(R^{\prime}\right)$. To extend this result to general monotonic transformations, we simply apply the "unilateral monotonic transformation step" iteratively for all agents $i \in N$.

Note that we did not use any restrictions on hospitals' preferences in the proof of Theorem 1. Hence, Theorem 1 is valid for the general preference domain, but also applies to our standard domain of matching markets where hospitals always have substitutable preferences. In addition, Theorem 1 is valid for any other non-empty preference domain for which the stable correspondence is well-defined (i.e., non-empty).

Theorem 2. If solution $\varphi$ is a Pareto efficient, individually rational, and monotonic correspondence, then $\varphi \supseteq S$, i.e., for all $R \in \mathcal{R}, \varphi(R) \supseteq S(R)$.

We give a short outline of the proof of Theorem 2 .

We start with a stable allocation $A$ for matching market $R$. In Step 1 we change doctors' preferences at $R$ and obtain a preference profile $R^{\prime} \in \mathcal{R}$ such that $R$ is a monotonic transformation of $R^{\prime}$ (and vice versa). In Step 2 we change hospitals' preferences at $R^{\prime}$ and obtain a substitutable preference profile $\bar{R}$ such that $R^{\prime}$ is a monotonic transformation of $\bar{R}$. Then, $R$ is also a monotonic transformation of $\bar{R}$. In Step 3 we show that $A$ is stable for matching market $\bar{R}$. In Step 4 we identify $A$ to be the only Pareto efficient and individual rational allocation for matching market $\bar{R}$. Hence, $A \in \varphi(\bar{R})$. Finally, since $\varphi$ is monotonic and $R$ is a monotonic transformation of $\bar{R}, A \in \varphi(R)$ (Step 5).

Proof: Let $\varphi$ be a Pareto efficient, individually rational, and monotonic correspondence. Consider a matching market $R \in \mathcal{R}$ and a stable set of contracts $A \in S(R)$. We need to show that $A \in \varphi(R)$.

Step 1: Transforming doctors' preferences

Let $D^{\prime} \subseteq D$ denote the set of doctors $d^{\prime}$ for whom there exists a contract $x_{d^{\prime}} \in X_{d^{\prime}}$ with $A_{d^{\prime}} P_{d^{\prime}} x_{d^{\prime}} P_{d^{\prime}} \emptyset$. 
For each doctor $d^{\prime} \in D^{\prime}$ we define $R_{d^{\prime}}^{\prime}$ by moving $\emptyset$ just below $A_{d^{\prime}}$ while not changing preferences over contracts in $X_{d^{\prime}}$, i.e., $R_{d^{\prime}}^{\prime}$ is such that

(i) $A_{d^{\prime}} P_{d^{\prime}}^{\prime} \emptyset$,

(ii) for no $\hat{x}_{d^{\prime}} \in X_{d^{\prime}}, A_{d^{\prime}} P_{d^{\prime}}^{\prime} \hat{x}_{d^{\prime}} P_{d^{\prime}}^{\prime} \emptyset$, and

(iii) for all $\bar{x}_{d^{\prime}}, \tilde{x}_{d^{\prime}} \in X_{d^{\prime}}, \bar{x}_{d^{\prime}} P_{d^{\prime}}^{\prime} \tilde{x}_{d^{\prime}}$ if and only if $\bar{x}_{d^{\prime}} P_{d^{\prime}} \tilde{x}_{d^{\prime}}$.

For all $i \in N \backslash D^{\prime}, R_{i}^{\prime}:=R_{i}$. Hence, we obtain matching market $R^{\prime}$ from $R$ by transforming preferences of doctors $d^{\prime} \in D^{\prime}$ according to (i)-(iii). Note that after transforming the doctors' preferences, hospitals' preferences are still substitutable. Also, note that for all $i \in N, L_{i}\left(R_{i}^{\prime}, A\right)=L_{i}\left(R_{i}, A\right)$. Hence, $R$ is a monotonic transformation of $R^{\prime}$ at $A$ and $R^{\prime}$ is a monotonic transformation of $R$ at $A$. By Theorem 1 and $R^{\prime} \in M T(R, A), A$ is a stable allocation for matching market $R^{\prime}$, i.e., $A \in S\left(R^{\prime}\right)$. Particularly, $A$ is individually rational for matching market $R^{\prime}$.

Step 2: Transforming hospitals' preferences

Recall that for all $h \in H, R_{h}^{\prime}=R_{h}$. To simplify notation, we will use for all $h \in H, C_{h}$ instead of $C_{h}^{\prime}$.

Let $\hat{H} \subseteq H$ denote the set of hospitals $\hat{h}$ for which there exists a contract $x_{\hat{h}},\left\{x_{\hat{h}}\right\} \in \mathcal{X}_{\hat{h}}$, and a set of contracts $Y \in \mathcal{X}_{\hat{h}}$ with $C_{\hat{h}}\left(A \cup\left\{x_{\hat{h}}\right\}\right)=A_{\hat{h}}$ and $\left(Y \cup\left\{x_{\hat{h}}\right\}\right) P_{\hat{h}} Y$. For any $x_{\hat{h}}$ such that $C_{\hat{h}}\left(A \cup\left\{x_{\hat{h}}\right\}\right)=A_{\hat{h}}$ define $\mathcal{Y}\left(x_{\hat{h}}, R_{\hat{h}}\right):=\left\{Y \in \mathcal{X}_{\hat{h}} \mid\left(Y \cup\left\{x_{\hat{h}}\right\}\right) P_{\hat{h}} Y\right\}$.

For each hospital $\hat{h} \in \hat{H}$ we fix some contract $x_{\hat{h}}$ such that $C_{\hat{h}}\left(A \cup\left\{x_{\hat{h}}\right\}\right)=A_{\hat{h}}$ and $\mathcal{Y}\left(x_{\hat{h}}, R_{\hat{h}}\right) \neq \emptyset$. Then, define $\hat{R}_{\hat{h}}$ as follows. For all $Y \in \mathcal{Y}\left(x_{\hat{h}}, R_{\hat{h}}\right)$ we move $Y$ just above $Y \cup\left\{x_{\hat{h}}\right\}$ while not changing preferences over sets of contracts in $\mathcal{X}_{\hat{h}} \backslash \mathcal{Y}\left(x_{\hat{h}}, R_{\hat{h}}\right)$, i.e., $\hat{R}_{\hat{h}}$ is such that for all $Y \in \mathcal{Y}\left(x_{\hat{h}}, R_{\hat{h}}\right)$,

(i) $Y \hat{P}_{\hat{h}}\left(Y \cup\left\{x_{\hat{h}}\right\}\right)$,

(ii) for no $Z \in \mathcal{X}_{\hat{h}}, Y \hat{P}_{\hat{h}} Z \hat{P}_{\hat{h}} Y \cup\left\{x_{\hat{h}}\right\}$, and

(iii) for all $\bar{Z}_{\hat{h}}, \tilde{Z}_{\hat{h}} \in \mathcal{X}_{\hat{h}} \backslash \mathcal{Y}\left(x_{\hat{h}}, R_{\hat{h}}\right), \bar{Z}_{\hat{h}} \hat{P}_{\hat{h}} \tilde{Z}_{\hat{h}}$ if and only if $\bar{Z}_{\hat{h}} P_{\hat{h}} \tilde{Z}_{\hat{h}}$.

Denote the associated choice correspondence for $\hat{h} \in \hat{H}$ at $\hat{R}$ by $\hat{C}_{\hat{h}}$. Before proving substitutability of $\hat{R}_{\hat{h}}$, we prove the following property of $\hat{C}_{\hat{h}}$. For all $Z \in \mathcal{X}_{\hat{h}}$,

(a) $\hat{C}_{\hat{h}}(Z)=C_{\hat{h}}\left(Z \cup\left\{x_{\hat{h}}\right\}\right) \backslash\left\{x_{\hat{h}}\right\}$,

which means that the set of chosen contracts at the new preference relation $\hat{R}_{\hat{h}}$ is obtained from the set of chosen contracts at the original preference relation $R_{\hat{h}}^{\prime}\left(=R_{\hat{h}}\right)$ by deleting contract $x_{\hat{h}}$ (in case $x_{\hat{h}}$ was chosen).

Step 2.1: Proof of (a)

If $x_{\hat{h}} \notin C_{\hat{h}}\left(Z \cup\left\{x_{\hat{h}}\right\}\right)$, then $C_{\hat{h}}\left(Z \cup\left\{x_{\hat{h}}\right\}\right) \subseteq Z$ and $C_{\hat{h}}(Z)=C_{\hat{h}}\left(Z \cup\left\{x_{\hat{h}}\right\}\right)$. Furthermore, $x_{\hat{h}} \notin C_{\hat{h}}\left(Z \cup\left\{x_{\hat{h}}\right\}\right)$ implies that $C_{\hat{h}}\left(Z \cup\left\{x_{\hat{h}}\right\}\right) \in \mathcal{X}_{\hat{h}} \backslash \mathcal{Y}\left(x_{\hat{h}}, R_{\hat{h}}\right)$. Then, (ii) and (iii) in the definition of $\hat{R}_{\hat{h}}$ imply that $\hat{C}_{\hat{h}}\left(Z \cup\left\{x_{\hat{h}}\right\}\right)=C_{\hat{h}}\left(Z \cup\left\{x_{\hat{h}}\right\}\right)$. Since $\hat{C}_{\hat{h}}\left(Z \cup\left\{x_{\hat{h}}\right\}\right) \subseteq Z$, $\hat{C}_{\hat{h}}(Z)=\hat{C}_{\hat{h}}\left(Z \cup\left\{x_{\hat{h}}\right\}\right)$. Thus, $\hat{C}_{\hat{h}}(Z)=\hat{C}_{\hat{h}}\left(Z \cup\left\{x_{\hat{h}}\right\}\right)=C_{\hat{h}}\left(Z \cup\left\{x_{\hat{h}}\right\}\right)=C_{\hat{h}}\left(Z \cup\left\{x_{\hat{h}}\right\}\right) \backslash\left\{x_{\hat{h}}\right\}$.

If $x_{\hat{h}} \in C_{\hat{h}}\left(Z \cup\left\{x_{\hat{h}}\right\}\right)$, then $C_{\hat{h}}\left(Z \cup\left\{x_{\hat{h}}\right\}\right) \backslash\left\{x_{\hat{h}}\right\} \in \mathcal{Y}\left(x_{\hat{h}}, R_{\hat{h}}\right)$. This together with (ii) and (iii) in the definition of $\hat{R}_{\hat{h}}$ implies that $\hat{C}_{\hat{h}}\left(Z \cup\left\{x_{\hat{h}}\right\}\right)=C_{\hat{h}}\left(Z \cup\left\{x_{\hat{h}}\right\}\right) \backslash\left\{x_{\hat{h}}\right\}$. Since $\hat{C}_{\hat{h}}\left(Z \cup\left\{x_{\hat{h}}\right\}\right) \subseteq Z, \hat{C}_{\hat{h}}(Z)=\hat{C}_{\hat{h}}\left(Z \cup\left\{x_{\hat{h}}\right\}\right)$. Thus, $\hat{C}_{\hat{h}}(Z)=C_{\hat{h}}\left(Z \cup\left\{x_{\hat{h}}\right\}\right) \backslash\left\{x_{\hat{h}}\right\}$. 


\section{Step 2.2: Proof of Substitutability}

Recall that since preferences $R_{h}$ are substitutable, we have for all $h \in H$,

(SUB) for all $X^{\prime} \subseteq Y^{\prime} \in \mathcal{X}_{h}, X^{\prime} \cap C_{h}\left(Y^{\prime}\right) \subseteq C_{h}\left(X^{\prime}\right)$.

We have to show that for all $\hat{h} \in \hat{H}$,

( $\widehat{\text { SUB }})$ for all $X^{\prime \prime} \subseteq Y^{\prime \prime} \in \mathcal{X}_{\hat{h}}, X^{\prime \prime} \cap \hat{C}_{\hat{h}}\left(Y^{\prime \prime}\right) \subseteq \hat{C}_{\hat{h}}\left(X^{\prime \prime}\right)$.

Let $\hat{h} \in \hat{H}$ and $X^{\prime \prime} \subseteq Y^{\prime \prime} \in \mathcal{X}_{\hat{h}}$. Consider $X^{\prime} \subseteq Y^{\prime} \in \mathcal{X}_{\hat{h}}$ such that $X^{\prime} \equiv\left(X^{\prime \prime} \cup\left\{x_{\hat{h}}\right\}\right)$ and $Y^{\prime} \equiv\left(Y^{\prime \prime} \cup\left\{x_{\hat{h}}\right\}\right)$. Then we have

$$
\begin{array}{ccc} 
& X^{\prime} \cap C_{\hat{h}}\left(Y^{\prime}\right) \\
\Rightarrow & \left(X^{\prime \prime} \cup\left\{x_{\hat{h}}\right\}\right) \cap C_{\hat{h}}\left(Y^{\prime \prime} \cup\left\{x_{\hat{h}}\right\}\right) \\
\Rightarrow & {\left[\left(X^{\prime \prime} \cup\left\{x_{\hat{h}}\right\}\right) \cap C_{\hat{h}}\left(Y^{\prime \prime} \cup\left\{x_{\hat{h}}\right\}\right)\right] \backslash\left\{x_{\hat{h}}\right\}} \\
\Rightarrow & X^{\prime \prime} \cap\left[C_{\hat{h}}\left(Y^{\prime \prime} \cup\left\{x_{\hat{h}}\right\}\right)\right] \backslash\left\{x_{\hat{h}}\right\} \\
\stackrel{\text { (a) }}{\Rightarrow} & \multicolumn{2}{c}{X^{\prime \prime} \cap \hat{C}_{\hat{h}}\left(Y^{\prime \prime}\right)}
\end{array}
$$$$
\begin{array}{cc}
\text { (SUB) } & C_{\hat{h}}\left(X^{\prime}\right) \\
\subseteq & C_{\hat{h}}\left(X^{\prime \prime} \cup\left\{x_{\hat{h}}\right\}\right) \\
\subseteq & C_{\hat{h}}\left(X^{\prime \prime} \cup\left\{x_{\hat{h}}\right\}\right) \backslash\left\{x_{\hat{h}}\right\} \\
\subseteq & C_{\hat{h}}\left(X^{\prime \prime} \cup\left\{x_{\hat{h}}\right\}\right) \backslash\left\{x_{\hat{h}}\right\} \\
\subseteq & \hat{C}_{\hat{h}}\left(X^{\prime \prime}\right),
\end{array}
$$

which shows $(\widehat{S U B})$. For all $i \in N \backslash \hat{H}, \hat{R}_{i}:=R_{i}^{\prime}$. Hence, we obtain matching market $\hat{R}$ from $R^{\prime}$ by transforming preferences of hospitals $\hat{h} \in \hat{H}$ according to (i)-(iii). We have shown that hospitals' preferences are still substitutable after the transformation. Also, note that for all $i \in N \backslash \hat{H}, L_{i}\left(\hat{R}_{i}, A\right)=L_{i}\left(R_{i}^{\prime}, A\right)$ and for all $\hat{h} \in \hat{H}, L_{\hat{h}}\left(\hat{R}_{\hat{h}}, A\right) \subseteq$ $L_{\hat{h}}\left(R_{\hat{h}}^{\prime}, A\right)$. Hence, $R^{\prime}$ is a monotonic transformation of $\hat{R}$ at $A$. Since $R$ is a monotonic transformation of $R^{\prime}$ at $A$, it follows that $R$ is a monotonic transformation of $\hat{R}$ at $A$. Finally, for all $i \in N, C_{i}(A)=\hat{C}_{i}(A)=A_{i}$. Thus, $\hat{C}_{D}(A)=\hat{C}_{H}(A)=A$ and $A$ is individually rational for matching market $\hat{R}$.

We repeat the transformation of hospitals' preferences as long as there exists a hospital $\tilde{h}$, a contract $x_{\tilde{h}},\left\{x_{\tilde{h}}\right\} \in \mathcal{X}_{\tilde{h}}$, and a set of contracts $Y \in \mathcal{X}_{\tilde{h}}$ with $C_{\tilde{h}}\left(A \cup\left\{x_{\tilde{h}}\right\}\right)=A_{\tilde{h}}$ and $\left(Y \cup\left\{x_{\tilde{h}}\right\}\right) P_{\tilde{h}} Y$. Note that this process stops after finitely many iterations since the number of contracts $x_{\tilde{h}}$ such that there exists $Y \in \mathcal{X}_{\tilde{h}}$ with $C_{\tilde{h}}\left(A \cup\left\{x_{\tilde{h}}\right\}\right)=A_{\tilde{h}}$ and $\left(Y \cup\left\{x_{\tilde{h}}\right\}\right) P_{\tilde{h}} Y$ decreases by one in each iteration.

Step 2.3: Matching Market $\bar{R}$

Finally, the transformation of agents' preferences results in a matching market $\bar{R}$ and an associated profile of choice correspondences $\left(\bar{C}_{i}\right)_{i \in N}$. Recall that doctors' preferences at $\bar{R}$ are the same as in $R^{\prime}$. The properties of $\hat{R}$ in each iteration induce that at $\bar{R}$ hospitals' preferences are substitutable, $R$ is a monotonic transformation of $\bar{R}$ at $A$, and $\bar{C}_{D}(A)=\bar{C}_{H}(A)=A$, i.e., $A$ is individually rational for matching market $\bar{R}$. Furthermore, for all hospitals $\bar{h}$ and contracts $x_{\bar{h}} \in \mathcal{X}_{\bar{h}}$ such that $C_{\bar{h}}\left(A \cup\left\{x_{\bar{h}}\right\}\right)=A_{\bar{h}}$, we have that

(b) for all $Y \in \mathcal{X}_{\bar{h}}$ such that $x_{\bar{h}} \notin Y, Y \bar{P}_{\bar{h}}\left(Y \cup\left\{x_{\bar{h}}\right\}\right)$.

Next we prove $A$ is a stable allocation for matching market $\bar{R}$.

Step 3: Proof that $A \in S(\bar{R})$

Suppose, by contradiction, that $A \notin S(\bar{R})$. Then, there exists a hospital $\bar{h}$ and a set of contracts $\bar{X} \in \mathcal{X}_{\bar{h}}, \bar{X} \neq \bar{C}_{\bar{h}}(A)$, such that $\bar{X}=\bar{C}_{\bar{h}}(A \cup \bar{X}) \subseteq \bar{C}_{D}(A \cup \bar{X})$. Since, $\bar{C}_{D}(A \cup$ $\bar{X})=C_{D}(A \cup \bar{X}), \bar{X} \subseteq C_{D}(A \cup \bar{X})$ (recall how doctors' preferences were transformed). 
Thus, $A \in S(R)$ implies $\bar{X} \neq C_{\bar{h}}(A \cup \bar{X})=A$. Hence, for some $\bar{d} \in D$ and $\bar{x}_{\bar{d}} \in \bar{X} \backslash A$, $\bar{x}_{\bar{d}} \bar{P}_{\bar{d}} A_{\bar{d}}$. By the stability of $A$ at matching market $R, C_{\bar{h}}\left(A \cup\left\{\bar{x}_{\bar{d}}\right\}\right)=A_{\bar{h}}$. Hence, for $\bar{X}_{\bar{h}} \in \mathcal{X}_{\bar{h}}$ by (b), $\left(\bar{X}_{\bar{h}} \backslash\left\{\bar{x}_{\bar{d}}\right\}\right) \bar{P}_{\bar{h}} \bar{X}_{\bar{h}}$. Since $\left(\bar{X}_{\bar{h}} \backslash\left\{\bar{x}_{\bar{d}}\right\}\right) \subseteq\left(A_{\bar{h}} \cup \bar{X}_{\bar{h}}\right)$, this constitutes a contradiction to $\bar{C}_{\bar{h}}(A \cup \bar{X})=\bar{X}$. Hence, $A \in S(\bar{R})$.

Since $A \in S(\bar{R})$, it follows that $A$ is Pareto efficient for matching market $\bar{R}$. Next, we show that allocation $A$ is the only Pareto efficient and individually rational allocation for matching market $\bar{R}$.

Step 4: Proof that $A$ is uniquely Pareto efficient and individually rational for $\bar{R}$ Suppose, by contradiction, that $\bar{A} \in \mathcal{A}, \bar{A} \neq A$, is Pareto efficient and individually rational for matching market $\bar{R}$.

Suppose there exists $\bar{d} \in D$ such that $\bar{A}_{\bar{d}} \bar{P}_{\bar{d}} A_{\bar{d}}$. By the transformation of doctors' preferences, $\bar{A}_{\bar{d}} P_{\bar{d}} A_{\bar{d}}$ and $\bar{A}_{\bar{d}} \neq \emptyset$. Let $\bar{h}$ be the hospital corresponding to $\bar{A}_{\bar{d}}$. Then, since $A \in S(R), C_{\bar{h}}\left(A \cup \bar{A}_{\bar{d}}\right)=A_{\bar{h}}$ and by (b), $\left(\bar{A}_{\bar{h}} \backslash\left\{\bar{A}_{\bar{d}}\right\}\right) \bar{P}_{\bar{h}} \bar{A}_{\bar{h}}$. Thus, in contradiction to individual rationality of $\bar{A}$ for $\bar{h}$ at matching market $\bar{R}, \bar{C}_{\bar{h}}(\bar{A}) \neq \bar{A}_{\bar{h}}$. Hence, for all $d \in D$, $A_{d} \bar{R}_{d} \bar{A}_{d}$.

Suppose there exists $\bar{h} \in H$ such that $\bar{A}_{\bar{h}} \bar{P}_{\bar{h}} A_{\bar{h}}$. Since $A \in S(\bar{R})$, there exists $\bar{d} \in D$ such that $\bar{A}_{\bar{d}} \in \bar{A}$ and $A_{\bar{d}} \bar{P}_{\bar{d}} \bar{A}_{\bar{d}}$. Thus, by the transformation of doctors' preferences $\emptyset \bar{R}_{\bar{d}} \bar{A}_{\bar{d}}$. If $\emptyset \bar{P}_{\bar{d}} \bar{A}_{\bar{d}}$, then we have an immediate contradiction to individual rationality of $\bar{A}$ for $\bar{d}$ at matching market $\bar{R}$. Hence, for all $d \in \bar{D}$ such that $\bar{A}_{\bar{d}} \in \bar{A}$ and $A_{d} \bar{P}_{d} \bar{A}_{d}$, $\bar{A}_{\bar{d}}=\emptyset$. Then, for all $d \in \bar{D}$ such that $\bar{A}_{\bar{d}} \in \bar{A}$ and $\bar{A}_{\bar{d}} \neq \emptyset, \bar{A}_{d} \bar{R}_{d} A_{d}$. This, together with $\bar{A}_{\bar{h}} \bar{P}_{\bar{h}} A_{\bar{h}}$, constitutes a contradiction to the stability of $\bar{A}$ at matching market $\bar{R}$. Hence, for all $h \in H, A_{h} \bar{R}_{h} \bar{A}_{h}$.

To summarize, for all $i \in N, A_{i} \bar{R}_{i} \bar{A}_{i}$. Since $A \neq \bar{A}$, for some $j \in N, A_{j} \bar{P}_{j} \bar{A}_{j}$. These facts establish a contradiction to the assumption that $\bar{A}$ is Pareto efficient.

Step 5: $A \in \varphi(R)$

Since $A$ is the unique individually rational and Pareto efficient allocation for matching market $\bar{R}$ it follows that $\varphi(\bar{R})=\{A\}$. Finally, since $\varphi$ is a monotonic solution and $R$ is a monotonic transformation of $\bar{R}$ at $A, A \in \varphi(R)$.

Note that in the proof of Theorem 2 we assume hospitals' preferences to be substitutable. Not assuming any restrictions on hospitals' preferences would in fact simplify the proof considerably since in the construction of preference profile $\hat{R}$ (and finally $\bar{R}$ ) in the proof of Theorem 2 special care has to be taken to guarantee that hospitals' preferences at $\hat{R}$ (and finally $\bar{R}$ ) are again substitutable.

In addition, Theorem 2 is valid for any other non-empty preference domain for which the stable correspondence is well-defined and for which the domain is closed with respect to the monotonic transformations needed in the proof: for instance, for college admissions problems, Kara and Sönmez (1997) prove the equivalent of Theorem 2 for the domain of responsive preferences that is a strict subdomain of the domain of substitutable preferences.

An immediate implication of Theorem 2 is that no strict selection from the stable correspondence is monotonic.

Corollary 1. Let $\varphi \subseteq S$, i.e., for all $R \in \mathcal{R}, \varphi(R) \subseteq S(R)$. If $\varphi$ is monotonic, then $\varphi=S$. 
Particularly, the well-defined (see Hatfield and Milgrom, 2005, Theorem 4) correspondence that always chooses the stable allocation that is most preferred by the doctors (alternatively, the correspondence that always chooses the stable allocation that is most preferred by the hospitals) is not monotonic and therefore not Nash implementable. Next, one can ask how to extend a non-monotonic correspondence in such a way that it becomes monotonic. Following Sen (1995) and Thomson (1999), we define the minimal monotonic extension of any correspondence $\psi$ as the smallest monotonic correspondence containing $\psi$, i.e.,

$$
m m e(\psi) \equiv \bigcap\{\phi \mid \psi \subseteq \phi, \text { where } \phi \text { is monotonic }\} .
$$

Hence, the stable correspondence is the minimal monotonic extension of any of its subcorrespondences.

Finally, we remark that a stronger version of Theorem 2 holds. Instead of requiring $\varphi(R)$ to only contain Pareto efficient, individual rational allocations, it suffices to assume that there is at least one Pareto efficient, individual rational allocation in $\varphi(R)$ for any $R \in \mathcal{R}$ (see also Theorems 3 and 5 in Kara, 1996).

\subsection{Nash Implementation}

A mechanism (for the described implementation environment) is a pair $(M, g)$, where $M:=\prod_{i \in N} M_{i}$ denotes a set of message or strategy profiles and $g: M \longrightarrow \mathcal{A}$ the so-called outcome function. The outcome function assigns to each strategy profile an allocation in $\mathcal{A}$. Since $g$ contains all relevant information, we identify a mechanism with its outcome function. A mechanism $g$ together with a preference profile $R$ induces a non-cooperative game in strategic form, denoted by $\Gamma(g, R)$, as follows. Each strategy profile $m \in M$ is mapped to an allocation $g(m) \in \mathcal{A}$. These outcomes of the game are then evaluated using the agents' preferences at $R$. Note that the fact that preferences in our context are ordinal does not limit the game theoretical analysis: either use ordinal preferences at $R$ to compare outcomes or choose a utility representation $u: \mathcal{A} \longrightarrow \mathbb{R}^{n}(n=|N|)$ of agents' preferences at $R$ and define the payoffs in $\Gamma(g, R)$ by the composition $u \circ g: M \longrightarrow \mathbb{R}^{n}$.

Mechanism $g$ Nash implements solution $\varphi$ if for all $R \in \mathcal{R}$ we obtain $g(N E(\Gamma(g, R)))=$ $\varphi(R)$, where $N E(\cdot)$ denotes the Nash equilibrium correspondence. Hence, for a given preference profile $R$ and any allocation $A \in \varphi(R)$ there is a Nash equilibrium of the induced game $\Gamma(g, R)$, the outcome of which is $A$. Conversely, the outcome of any Nash equilibrium of $\Gamma(g, R)$ belongs to $\varphi(R)$. We say that a solution $\varphi$ is Nash implementable, if there exists a mechanism that Nash implements it.

Loosely speaking, a mechanism $g$ describes a list of rules for a game in strategic form. These rules are independent of the true preference profile. Then, for any possible set of agents, represented by their preference profile $R$, any (desired) allocation in $\varphi(R)$ can be achieved by strategic interaction in equilibrium, thus can be obtained in a non-cooperative fashion.

Maskin $(1977,1999)$ showed that a Nash implementable solution necessarily has to be monotonic. However, monotonicity is not a sufficient condition for implementability. Moore and Repullo (1990) provide a necessary and sufficient condition for Nash implementability of a solution. We show that the stable correspondence does satisfy this condition and hence can be implemented by a version of Maskin's (1999) mechanism (see Moore and Repullo, 1990, Appendix). 
For any agent $i \in N$, matching market $R \in \mathcal{R}$, and subset $\mathcal{A}^{\prime} \subseteq \mathcal{A}$ of allocations let $B_{i}\left(R, \mathcal{A}^{\prime}\right)$ denote the set of "best allocations for agent $i$ in $\mathcal{A}^{\prime}$ with respect to preference relation $R_{i}$ ", i.e., $B_{i}\left(R, \mathcal{A}^{\prime}\right):=\left\{A \in \mathcal{A}^{\prime} \mid A_{i} R_{i} A_{i}^{\prime}\right.$ for all $\left.A^{\prime} \in \mathcal{A}^{\prime}\right\}$. Note that if $A \in B_{i}\left(R, \mathcal{A}^{\prime}\right)$ and $A^{\prime} \in \mathcal{A}^{\prime}$ is such that $A_{i}=A_{i}^{\prime}$ then $A^{\prime} \in B_{i}\left(R, \mathcal{A}^{\prime}\right)$. That means that all best allocations in $B_{i}\left(R, \mathcal{A}^{\prime}\right)$ specify the same (set of) contract(s) for agent $i$. Note that $\mathcal{A}^{\prime} \neq \emptyset$ implies $B_{i}\left(R, \mathcal{A}^{\prime}\right) \neq \emptyset$.

Definition 1. Condition $\mu$ in Moore and Repullo (1990)

A solution $\varphi: \mathcal{R} \Longrightarrow \mathcal{A}$ satisfies condition $\mu$, if there exists a set $\mathcal{B} \subseteq \mathcal{A}$ and for each $i \in N, R \in \mathcal{R}$ and $A \in \varphi(R)$ there is a set $T_{i}(R, A) \subseteq \mathcal{B}$ with $A \in B_{i}\left(R, T_{i}(R, A)\right)$ such that for each $\bar{R} \in \mathcal{R}$ and $j \in N$ the following three conditions are satisfied:

( $\mu 1) \quad A \in \bigcap_{i \in N} B_{i}\left(\bar{R}, T_{i}(R, A)\right)$ implies $A \in \varphi(\bar{R})$,

$(\mu 2) A^{*} \in B_{j}\left(\bar{R}, T_{j}(R, A)\right) \cap \bigcap_{i \in N \backslash\{j\}} B_{i}(\bar{R}, \mathcal{B})$ implies $A^{*} \in \varphi(\bar{R})$,

$(\mu 3) A^{*} \in \bigcap_{i \in N} B_{i}(\bar{R}, \mathcal{B})$ implies $A^{*} \in \varphi(\bar{R})$.

Moore and Repullo (1990, Theorem 1) show that in the presence of three or more agents a solution $\varphi$ is Nash implementable if and only if it satisfies condition $\mu$.

Theorem 3. If $|N| \geq 3$, then the stable correspondence $S$ is Nash implementable.

Proof: We show that the stable correspondence $S$ satisfies condition $\mu$ (Definition 1), given $R \in \mathcal{R}$ and $A \in S(R)$, with $\mathcal{B}=\mathcal{A}$ and $T_{i}(R, A)=L_{i}\left(R_{i}, A\right)$. Let $\bar{R} \in \mathcal{R},\left(\bar{C}_{i}\right)_{i \in N}$ be the associated profile of choice correspondences, and $j \in N$. Then, condition $\mu$ reads as follows:

( $\mu 1) \quad A \in \bigcap_{i \in N} B_{i}\left(\bar{R}, L_{i}\left(R_{i}, A\right)\right)$ implies $A \in S(\bar{R})$,

$(\mu 2) \quad A^{*} \in B_{j}\left(\bar{R}, L_{j}\left(R_{j}, A\right)\right) \cap \bigcap_{i \in N \backslash\{j\}} B_{i}(\bar{R}, \mathcal{A})$ implies $A^{*} \in S(\bar{R})$,

$(\mu 3) \quad A^{*} \in \bigcap_{i \in N} B_{i}(\bar{R}, \mathcal{A})$ implies $A^{*} \in S(\bar{R})$.

Step 1: $S$ satisfies condition $(\mu 1)$

Note that by the strictness of agents' preferences the following statements are equivalent

(i) $A \in \bigcap_{i \in N} B_{i}\left(\bar{R}, L_{i}\left(R_{i}, A\right)\right)$

(ii) $\{A\}=\bigcap_{i \in N} B_{i}\left(\bar{R}, L_{i}\left(R_{i}, A\right)\right)$,

(iii) $\bar{R} \in M T(R, A)$. 
Hence, condition ( $\mu 1$ ) is equivalent to monotonicity (see also Moore and Repullo, 1990). Thus, Theorem 1 implies that $S$ satisfies condition $(\mu 1)$.

Step 2: $A^{*} \in B_{i}(\bar{R}, \mathcal{A})$ implies for all $X^{\prime} \subseteq X, \bar{C}_{i}\left(X^{\prime} \cup A^{*}\right)=\bar{C}_{i}\left(A^{*}\right)=A_{i}^{*}$

Let $A^{*} \in B_{i}(\bar{R}, \mathcal{A})$. Since $B_{i}(\bar{R}, \mathcal{A})$ denotes the set of best allocations for agent $i$ among all possible allocations $\mathcal{A}$ with respect to preference relation $\bar{R}_{i}$, agent $i$ must receive the best possible (set of) contract(s) in $X$. Hence, $\bar{C}_{i}(X)=\bar{C}_{i}\left(A^{*}\right)=A_{i}^{*}$ and for all $X^{\prime} \subseteq X$, $\bar{C}_{i}\left(X^{\prime} \cup A^{*}\right)=\bar{C}_{i}\left(A^{*}\right)$.

Step 3: $A^{*} \in B_{i}\left(\bar{R}, L_{i}\left(R_{i}, A\right)\right)$ implies $\bar{C}_{i}\left(A^{*}\right)=A_{i}^{*}$

Let $i=d \in D$ and $A^{*} \in B_{d}\left(\bar{R}, L_{d}\left(R_{d}, A\right)\right)$. Let $A^{\prime} \in \mathcal{A}$ be such that $A_{d}^{\prime}=\emptyset$ and for all $i \in N \backslash\{d\}, A_{i}^{\prime}=A_{i}^{*}$. Then, $A \in S(R)$ (IR) implies that $A_{d} R_{d} \emptyset$ and $A^{\prime} \in L_{d}\left(R_{d}, A\right)$. Thus, since $A^{*}$ is a best allocation for doctor $d$ in $L_{d}\left(R_{d}, A\right)$ with respect to preference relation $\bar{R}_{d}$, either $A_{d}^{*} \bar{P}_{d} \emptyset$ or $A_{d}^{*}=\emptyset$. Thus, $\bar{C}_{d}\left(A^{*}\right)=A_{d}^{*}$.

Let $i=h \in H$ and $A^{*} \in B_{h}\left(\bar{R}, L_{h}\left(R_{h}, A\right)\right)$. Let $X^{\prime} \subseteq A_{h}$ and $A^{\prime} \in \mathcal{A}$ be such that $A_{h}^{\prime}=X^{\prime}$ and for all $i \in N \backslash\{h\}, A_{i}^{\prime}=A_{i}^{*}$. Then, $A \in S(R)$ (IR) implies that $A_{h} R_{h} X^{\prime}$ and $A^{\prime} \in L_{h}\left(R_{h}, A\right)$. Thus, since $A^{*}$ is a best allocation for hospital $h$ in $L_{h}\left(R_{h}, A\right)$ with respect to preference relation $\bar{R}_{h}$, either $A_{h}^{*} \bar{P}_{h} X^{\prime}$ or $A_{d}^{*}=X^{\prime}$. Thus, $\bar{C}_{d}\left(A^{*}\right)=A_{d}^{*}$.

Step 4: The assumptions in ( $\mu 2)$ as well as the assumptions in ( $\mu 3)$ imply (IR), i.e., $\bar{C}_{D}\left(A^{*}\right)=\bar{C}_{H}\left(A^{*}\right)=A^{*}$

Let $A^{*}$ be either as required in $(\mu 2)$ or $(\mu 3)$. Hence, for all $i \in N$ either $A^{*} \in B_{i}(\bar{R}, \mathcal{A})$ or $A^{*} \in B_{i}\left(\bar{R}, L_{i}\left(R_{i}, A\right)\right)$. By Steps 2 and 3 , for all $i \in N, \bar{C}_{i}\left(A^{*}\right)=A_{i}^{*}$. Hence $\bar{C}_{D}\left(A^{*}\right)=$ $\bar{C}_{H}\left(A^{*}\right)=A^{*}$ and $A^{*}$ satisfies (IR) in the definition of stability.

Step 5: The assumptions in ( $\mu 2)$ as well as the assumptions in ( $\mu 3)$ imply (NB), i.e., there is no hospital $h$ and set of contracts $X^{\prime} \in \mathcal{X}_{h}, X^{\prime} \neq \bar{C}_{h}\left(A^{*}\right)$, such that $X^{\prime}=$ $\bar{C}_{h}\left(A^{*} \cup X^{\prime}\right) \subseteq \bar{C}_{D}\left(A^{*} \cup X^{\prime}\right)$

Let $A^{*}$ be either as required in $(\mu 2)$ or $(\mu 3)$. Let $h \in H$ be such that $A^{*} \in B_{h}(\bar{R}, \mathcal{A})$ and $X^{\prime} \in \mathcal{X}_{h}$ with $X^{\prime} \neq \bar{C}_{h}\left(A^{*}\right)$ such that $X^{\prime}=\bar{C}_{h}\left(A^{*} \cup X^{\prime}\right)\left[\subseteq \bar{C}_{D}\left(A^{*} \cup X^{\prime}\right)\right]$. By Step 2, $\bar{C}_{h}\left(A^{*} \cup X^{\prime}\right)=\bar{C}_{h}\left(A^{*}\right)$, which implies $X^{\prime}=\bar{C}_{h}\left(A^{*}\right)$ - a contradiction.

Let $h \in H$ such that $A^{*} \in B_{h}\left(\bar{R}, L_{h}\left(R_{h}, A\right)\right)$ and $X^{\prime} \in \mathcal{X}_{h}$ with $X^{\prime} \neq \bar{C}_{h}\left(A^{*}\right)$ such that $X^{\prime}=\bar{C}_{h}\left(A^{*} \cup X^{\prime}\right) \subseteq \bar{C}_{D}\left(A^{*} \cup X^{\prime}\right)$. Then, by Step 2, for all $i \in N \backslash\{h\}, \bar{C}_{i}\left(X^{\prime} \cup A^{*}\right)=$ $\bar{C}_{i}\left(A^{*}\right)=A_{i}^{*}$. Thus, $\bar{C}_{D}\left(A^{*} \cup X^{\prime}\right)=\bar{C}_{D}\left(A^{*}\right)=A_{h}^{*}$ and therefore $X^{\prime} \subseteq A_{h}^{*}$. By Step 3, $\bar{C}_{h}\left(A^{*}\right)=A_{h}^{*}$. Hence, $\bar{C}_{h}\left(A^{*} \cup X^{\prime}\right)=\bar{C}_{h}\left(A^{*}\right)=A_{h}^{*}$, which implies $X^{\prime}=\bar{C}_{h}\left(A^{*}\right)$ - a contradiction.

Steps 4 and 5 together show $A^{*} \in S(\bar{R})$ given the assumptions in $(\mu 2)$ or $(\mu 3)$.

Two remarks on Theorem 3 are in order:

First, we did not use any restrictions on hospitals' preferences in the proof of Theorem 3. Similarly as before (Theorem 1) we used substitutability of hospitals' preferences only to guarantee the existence of stable matchings and Theorem 3 is valid for any other non-empty preference domain for which the stable correspondence is well-defined.

Second, in the implementing mechanism $g$ in Moore and Repullo (1990) the mathematical objects $\mathcal{B}$ and $T_{i}(R, A)$ in Condition $\mu$ have an intuitive interpretation. The allocations in $\mathcal{B}$ are exactly those that can result from strategic interaction in the mechanism. For any $A \in S(R)$ let $\bar{m}$ be an equilibrium with $g(\bar{m})=A$. Then $T_{i}(R, A)$ contains those allocations that agent $i$ can "enforce" by unilaterally deviating from $\bar{m}$. Clearly, with $T_{i}(R, A)=L_{i}\left(R_{i}, A\right)$, neither agent is willing to deviate. The fact that $\mathcal{B}$ can be set 
to $\mathcal{A}$ shows that in the mechanism every allocation can be an outcome of the mechanism, i.e., it can be achieved through strategic interaction.

Theorem 3 holds as long as there are at least three agents in the market. Let us briefly turn to the case in which there is exactly one doctor and one hospital. Hence the matching with contracts model is interpreted as choosing an appropriate contract between these two agents. We get a negative implementation result as long as we consider the whole set of possible preference profiles.

Theorem 4. Suppose $|D|=|H|=1$, then $S(R)$ is the set of all Pareto efficient, individually rational allocations. Moreover, $S$ is not Nash implementable.

Proof: The first statement directly follows from the stability conditions (IR) and (NB). The set of allocations can be identified with $X \cup\{\emptyset\}$ and each $\mathcal{R}_{i}$ consists of all strict orderings over $X \cup\{\emptyset\}$. Substitutability is not a restriction in this case.

Note that $S$ is not dictatorial ${ }^{5}$. Non-implementability of the stable correspondence then follows from Theorem 1 in Maskin (1999) (see also Hurwicz and Schmeidler, 1978), which states that in an environment with two agents and $\mathcal{R}$ containing all strict preference profiles over $\mathcal{A}$, a Pareto optimal solution $\varphi$ that is Nash implementable necessarily has to be dictatorial. Hence, as $S$ is Pareto efficient and not dictatorial, it cannot be Nash implementable.

Remark 1. Theorem 4 crucially relies on the fact that all strict preferences over allocations are contained in $\mathcal{R}$. However, in specific cases, one can obtain a positive implementation result for two agents. Suppose, for example, that $\mathcal{R}$ contains all preference profiles, in which the empty contract is always ranked least by the doctor as well as by the hospital. Thus, it is clear from the beginning that employment under any conditions is better than staying unemployed: a so-called bad outcome exists. As noted above, the stable correspondence collects all Pareto efficient, individually rational allocations. It is easy to see, that $S(\cdot)$ therefore satisfies "no veto power", which states that allocations that are maximal for $n-1$ agents at profile $R$ have to belong to $S(R) .{ }^{6}$ Following Moore and Repullo (1990), this implies a weaker condition called restricted veto power. Then, by Moore and Repullo (1990, Corollary 3), restricted veto power in connection with the existence of a bad outcome suffices to show that the stable correspondence is Nash implementable in the two-agent case. To sum, although the two agents may be fully informed about each other's preferences this knowledge cannot credibly be shared with a third party, as long as we have no restrictions on the set of possible preference profiles. However, if we can take employment for granted, there is a mechanism that implements the stable correspondence, which then achieves a Pareto efficient, individually rational allocation through strategic interaction.

\footnotetext{
${ }^{5}$ A solution $\varphi$ is dictatorial, if there exists $i \in N$ such that for all $R \in \mathcal{R}, A \in \varphi(R)$ implies for all $A^{\prime} \in \mathcal{A}, A R_{i} A^{\prime}$.

${ }^{6}$ For all $i \in N, R \in \mathcal{R}$, and $A \in \mathcal{A}, A \in \bigcap_{j \neq i} B_{j}(R, \mathcal{A})$ implies $A \in S(R)$.
} 


\section{Conclusion}

One of our main results is that in two-sided matching markets with contracts and more than two agents the stable correspondence is Nash implementable (Theorem 3). For two agent markets implementability can no longer be established (Theorem 4). Furthermore, we show that the stable correspondence is monotonic (Theorem 1) and minimal among all monotonic, Pareto efficient and individually rational solutions (Theorem 2). As matching markets with contracts comprise a large class of different types of matching markets our results extend previous Nash implementability results in more specific matching markets (e.g., marriage and college admissions markets as considered by Kara and Sönmez, 1996, 1997). This extension is twofold. First, agents can be matched under different contract conditions where previously only one contract per match was possible (e.g., "being matched" was the only available contract). Second, we extend the preference domain from responsive to substitutable preferences (an extension that is not trivial and might affect the structure of the set of stable allocations; see Martínez et al., 1991).

An alternative approach to show implementability of the stable correspondence $S$ is to show that it satisfies essential monotonicity ${ }^{7}$ which is a stronger condition than monotonicity. We could then obtain implementability of the stable correspondence by using Yamato's (1992) Theorem 2, which states (in our context) that Nash implementability and essential monotonicity are equivalent.

In contrast to Kara and Sönmez (1996, 1997), Sönmez (1996), or Ehlers (2004), who rely on Yamato's (1992) theorem, we apply Moore and Repullo's (1990) condition $\mu$ (Definition 1). Thus, instead of emphasizing that the stable correspondence satisfies a stronger version of monotonicity, we focus on a weak "no veto power" property that the stable correspondence satisfies and that together with monotonicity guarantees that stable allocations can be achieved through strategic interaction. ${ }^{8}$

More precisely, in view of condition $\mu$ as used in the proof of Theorem 3, we can interpret $(\mu 2)$ in the following way. Suppose we have a stable allocation $A$ at preference profile $R$. After a change in preferences from $R$ to $\bar{R}$ there is an allocation $A^{*}$, which is among the best possible allocation for all agents except agent $j$. Then, this property alone is not sufficient to identify $A^{*}$ as a stable allocation at $\bar{R}$, since agent $j$ (say $j \in D$ ) might prefer the empty contract to his contract in $A^{*}$ and is also able to enforce it. Since $A$ was a stable allocation (at $R$ ), the empty contract clearly appears in $L_{j}\left(R_{j}, A\right)$. So, condition $(\mu 2)$ says that agent $j$ cannot veto against $A^{*}$, if he gets his best contract (at $\left.\bar{R}\right)$ from $L_{j}\left(R_{j}, A\right)$ at $\bar{R}$. In fact, none of the agents except agent $j$ would want to block $A^{*}$ and stability enables agent $j$ in this situation to choose any contract he can enforce on his own. Hence, we mainly need the individual rationality property of stable allocations to satisfy $(\mu 2)$. Condition $(\mu 3)$ simply describes a unanimity condition stating that if there is an allocation that is best for all agents, then this allocation should be chosen. Clearly, such an allocation satisfies individual rationality and no-blocking. Thus, stability clearly implies the unanimity principle stated in $(\mu 3)$.

\footnotetext{
${ }^{7}$ Roughly speaking, an allocation $A^{\prime} \in L_{i}\left(R_{i}, A\right)$ is essential for agent $i$ at preference profile $R$ and allocation $A \in S(R)$ if there exists a profile $\hat{R}$ such that $A^{\prime} \in S(\hat{R})$ and $L_{i}\left(\hat{R}_{i}, A^{\prime}\right) \subseteq L_{i}\left(R_{i}, A\right)$. Denoting by $E_{i}(R, A)$ the set of essential allocations for $i$, the stable correspondence $S$ is essentially monotonic if the following holds: for all $A \in \mathcal{A}, R, \hat{R} \in \mathcal{R}, A \in S(R)$ and $E_{i}(R, A) \subseteq L_{i}(\hat{R}, A)$ imply $A \in S(\hat{R})$.

${ }^{8}$ A solution $\varphi: \mathcal{R} \Longrightarrow \mathcal{A}$ satisfies no veto power, if for all $i \in N$ we have that $A \in \bigcap_{j \neq i} B_{j}(R, \mathcal{A})$ implies $A \in \varphi(R)$, i.e., agent $i$ cannot veto $A$ if it is among the best allocations for any other agent.
} 


\section{A Well-Known Matching with Contracts Markets}

\section{Example 1. One-To-One Matching or Marriage Markets}

Gale and Shapley (1962) introduced one-to-one matching or marriage markets (see also Roth and Sotomayor, 1990, Part I). They demonstrated that stable matchings for one-toone matching markets always exist and at least one stable matching can be calculated by using the (now) famous deferred acceptance algorithm.

Using our matching with contracts framework, a one-to-one matching market consists of $D, H$, and $X=\{(d, h) \mid d \in D$ and $h \in H\}$. Each doctor $d \in D$ has a total (linear) order over $X_{d} \cup\{\emptyset\}=\{(d, h) \mid h \in H\} \cup\{\emptyset\}$. Each hospital $h \in H$ has a total (linear) order over the sets of feasible contracts $\mathcal{X}_{h}=\left\{X^{\prime} \mid X^{\prime} \subseteq\{(d, h) \mid d \in D\}\right\}$ that satisfies the following restriction: for all $X^{\prime} \in \mathcal{X}_{h},\left|X^{\prime}\right|>1, \emptyset P_{h} X^{\prime}$. Phrased differently, for all $X^{\prime} \subseteq X,\left|C_{h}\left(X^{\prime}\right)\right| \leq 1$, i.e., from any set of contracts $X^{\prime}$ a hospital $h$ either chooses the null contract or exactly one contract in $X^{\prime} \cap\{(d, h) \mid d \in D\}$. Note that hospitals' preferences as specified above are substitutable.

In the original model (Gale and Shapley, 1962) the set of feasible contracts per hospital is restricted to at most one contract and hospitals' preferences are defined as total (linear) orders over $\{(d, h) \mid d \in D\} \cup\{\emptyset\}$. In view of results concerning stability it does not matter whether one restricts the set of feasible contracts for hospitals or incorporates these restrictions into the hospitals' individual rationality constraints through their preferences.

\section{Example 2. Many-To-One Matching or College Admissions Markets}

Gale and Shapley (1962) first introduced many-to-one matching or college admissions markets. In their original model colleges are matched with sets of students, but colleges' preferences are only defined over individual students and not over sets of students. The college admissions problem where colleges have preferences over sets of students was first presented by Roth (1985) (see also Roth and Sotomayor, 1990, Part II). Roth (1985) demonstrated that contrary to the general belief, many-to-one matching markets cannot be reduced to one-to-one matching markets by reducing colleges' preferences to preferences over individual students. For instance, in contrast to one-to-one matching markets, stable matchings may not exist for many-to-one matching markets.

Using our matching with contracts framework, a many-to-one matching market consists of $D, H$, a quota vector $q=\left(q_{h}\right)_{h \in H}$ (for all $h \in H, q_{h}>0$ is a positive integer that represents the maximal number of doctors hospital $h$ can hire) and $X=\{(d, h) \mid d \in$ $D$ and $h \in H\}$. Each doctor $d \in D$ has a total (linear) order over $X_{d} \cup\{\emptyset\}=\{(d, h) \mid h \in$ $H\} \cup\{\emptyset\}$. Each hospital $h \in H$ has a total (linear) order over the sets of feasible contracts $\mathcal{X}_{h}=\left\{X^{\prime} \mid X^{\prime} \subseteq\{(d, h) \mid d \in D\}\right\}$ that satisfies the following restriction: for all $X^{\prime} \in \mathcal{X}_{h}$, $\left|X^{\prime}\right|>q_{h}, \emptyset P_{h} X^{\prime}$. Phrased differently, for all $X^{\prime} \subseteq X,\left|C_{h}\left(X^{\prime}\right)\right| \leq q_{h}$, i.e., from any set of contracts $X^{\prime}$ a hospital $h$ chooses at most $q_{h}$ contracts in $X^{\prime} \cap\{(d, h) \mid d \in D\}$. In addition, in order to express that hospitals' preferences over sets of doctors are linked in a plausible way to their preferences over individual doctors, we assume that hospitals' preferences are responsive (see Roth, 1985): Let $h \in H$. Then, (i) for all $X^{\prime} \subseteq\{(d, h) \mid d \in D\},\left|X^{\prime}\right|<q_{h}$, and all $\left(d^{\prime}, h\right) \notin X^{\prime}, X^{\prime} \cup\left\{\left(d^{\prime}, h\right)\right\} P_{h} X^{\prime}$ if and only if $\left\{\left(d^{\prime}, h\right)\right\} P_{h} \emptyset$ and (ii) for all $X^{\prime} \subseteq\{(d, h) \mid d \in D\},\left|X^{\prime}\right|<q_{h}$, and all $\left(d^{\prime}, h\right),\left(d^{\prime \prime}, h\right) \notin X^{\prime}, X^{\prime} \cup\left\{\left(d^{\prime}, h\right)\right\} P_{h} X^{\prime} \cup\left\{\left(d^{\prime \prime}, h\right)\right\}$ if and only if $\left\{\left(d^{\prime}, h\right)\right\} P_{h}\left\{\left(d^{\prime \prime}, h\right)\right\}$. Note that if a hospital's preferences are responsive then they are also substitutable. 
If for all $h \in H, q_{h}=1$, then the many-to-one and the one-to-one (see Example 1) matching model coincide. Similarly as in Example 1, in view of results concerning stability it does not matter whether one restricts the set of feasible contracts for hospitals by their quotas or incorporates these restrictions into the hospitals' individual rationality constraints through their preferences.

Example 3. Many-To-One Matching with Wages or Job Matching Markets In order to fit into the matching with contracts model, we discuss a discrete version without ties of Kelso and Crawford's (1982) job matching model. (Note that in Kelso and Crawford's (1982) original (discrete or continuous) model, ties between contracts are allowed.)

A many-to-one matching market with wages consists of $D, H$, a discrete set of wages $W \subset \mathbb{R}_{+}, X \subset D \times H \times W$, and corresponding projections $\mu_{D}$ and $\mu_{H}$ on $D$ and $H$, respectively. We assume that $W$ is such that agents have strict preferences. Each doctor $d \in D$ has a total (linear) order over $X_{d} \cup\{\emptyset\}=\{(d, h, w) \in X \mid h \in H, w \in W\} \cup\{\emptyset\}$ such that for two contracts $(d, h, w)$ and $\left(d, h, w^{\prime}\right)$, doctor $d$ (strictly) prefers $(d, h, w)$ to $\left(d, h, w^{\prime}\right)$ if and only if $w>w^{\prime}$. By $w_{(d, h)}$ we denote the smallest wage at which doctor $d$ (strictly) prefers to work at hospital $h$ over being unemployed, denoted by $\emptyset$. Each hospital $h \in H$ has a total (linear) order over the sets of feasible contracts $\mathcal{X}_{h}=\left\{X^{\prime} \subseteq \mu_{H}^{-1}(h) \mid\right.$ for all $d \in$ $\left.D,\left|X^{\prime} \cap \mu_{D}^{-1}(d)\right| \leq 1\right\}$ that are based on a "production" function $Y^{h}$ satisfying the following properties: (i) for all $D^{\prime} \subseteq D, Y^{h}\left(D^{\prime}\right) \in \mathbb{R}_{+}$represents the productivity (in monetary terms) of the set of doctors $D^{\prime}$ when employed together at hospital $h$, (ii) $Y^{h}(\emptyset)=0$ (no free lunch), and (iii) for all $D^{\prime} \subseteq D \backslash\left\{d^{\prime}\right\}, Y^{h}\left(D^{\prime} \cup d^{\prime}\right)-Y^{h}\left(D^{\prime}\right) \geq w_{\left(d^{\prime}, h\right)} \cdot{ }^{9}$ Assumption (iii) implies that in a Kelso and Crawford job matching market there are no unemployed doctors (at least not at a stable allocation), since hiring one more doctor at minimal wage always benefits the hospital. Finally, hospital $h$ (strictly) prefers $\bigcup_{d \in D^{\prime}}\left(d, h, w_{d}^{\prime}\right) \in \mathcal{X}_{h}$ to $\bigcup_{d \in D^{\prime \prime}}\left(d, h, w_{d}^{\prime}\right) \in \mathcal{X}_{h}$ if and only if $Y^{h}\left(D^{\prime}\right)-\sum_{d \in D^{\prime}} w_{d}>Y^{h}\left(D^{\prime \prime}\right)-\sum_{d \in D^{\prime \prime}} w_{d}$. Hatfield and Milgrom (2005, Theorem 2) show that Kelso and Crawford's "gross-substitutability" condition implies substitutability of hospitals' preferences.

\section{Example 4. Auction Markets}

A matching market with contracts can be interpreted as an auction market if there is only one hospital (the auctioneer) which auctions off a set of (real or abstract) objects. A contract for such a matching market specifies the set of objects an agent obtains at which price. We refer to Hatfield and Milgrom (2005) and Milgrom (2004) for further examples and references concerning the connection between matching with contracts and auctions.

\footnotetext{
${ }^{9}$ The last condition is a modeling convention since without (iii) a hospital could always employ a doctor whose marginal product is less than his/her minimal wage at zero wage with the agreement that he/she does not have to work.
} 


\section{References}

Alkan, A. and Gale, D. (2003): "Stable Schedule Matching under Revealed Preferences." Journal of Economic Theory, 112: 289-306.

Echenique, F. and Oviedo, J. (2006): "A Theory of Stability in Many-to-Many Matching Markets." Theoretical Economics, 1: 233-273.

Ehlers, L. (2004): "Monotonic and Implementable Solutions in Generalized Matching Problems." Journal of Economic Theory, 114: 358-369.

Gale, D. and Shapley, L. (1962): "College Admissions and the Stability of Marriage." American Mathematical Monthly, 69: 9-15.

Hatfield, J. W. and Milgrom, P. (2005): "Matching with Contracts." American Economic Review, 95(4): 913-935.

Hurwicz, L. and Schmeidler, D. (1978): "Construction of Outcome Functions Guaranteeing Existence and Pareto Optimality of Nash Equilibria." Econometrica, 46: 1447-74.

Jackson, M. O. (2001): "A Crash Course in Implementation Theory." Social Choice and Welfare, 18(3): 655-708.

Kara, T. (1996): Implementation in Matching Problems. Ph.D. thesis, University of Rochester.

Kara, T. and Sönmez, T. (1996): "Nash Implementation of Matching Rules." Journal of Economic Theory, 68: 425-439.

Kara, T. and Sönmez, T. (1997): "Implementation of College Admission Rules." Economic Theory, 9: 197-218.

Kelso, A. S. and Crawford, V. P. (1982): "Job Matching, Coalition Formation, and Gross Substitutes." Econometrica, 6: 1483-1504.

Martínez, R., Massó, J., Neme, A., and Oviedo, J. (1991): "Single Agents and the Set of Many-to-One Stable Matchings." Journal of Economic Theory, 91: 91-105.

Maskin, E. (1977): "Nash Equilibrium and Welfare Optimality." MIT Working Paper.

Maskin, E. (1999): "Nash Equilibrium and Welfare Optimality." Review of Economic Studies, 66: 23-38.

Maskin, E. and Sjöström, T. (2002): "Implementation Theory." In Handbook of Social Choice and Welfare, volume 1. North-Holland.

Milgrom, P. (2004): Putting Auction Theory to Work. Cambridge University Press, Cambridge.

Moore, J. and Repullo, R. (1990): "Nash Implementation: A full Characterization." Econometrica, 58(5): 1083-1099.

Roth, A. E. (1982): "The Economics of Matching: Stability and Incentives." Mathematics of Operations Research, 7: 617-628. 
Roth, A. E. (1984a): "The Evolution of the Labor Market for Medical Interns and Residents: a Case Study in Game Theory." Journal of Political Economy, 92: 991-1016.

Roth, A. E. (1984b): "Stability and Polarization of Interests in Job Matching." Econometrica, 52(1): 47-57.

Roth, A. E. (1985): "The College Admissions Problem Is Not Equivalent to the Marriage Problem." Journal of Economic Theory, 63(2): 277-288.

Roth, A. E. (1991): "A Natural Experiment in the Organization of Entry-Level Labor Markets: Regional Markets for New Physicians and Surgeons in the United Kingdom." American Economic Review, 81: 415-440.

Roth, A. E. and Sotomayor, M. A. (1990): Two-Sided Matching: A Study in GameTheoretic Modeling and Analysis. Cambridge University Press, Cambridge.

Roth, A. E. and Xing, X. (1994): "Jumping the Gun: Imperfections and Institutions Related to the Timing of Market Transactions." American Economic Review, 84: 9921044 .

Sen, A. (1995): "The Implementation of Social Choice Functions via Social Choice Correspondences: a general Formulation and a limited Result." Social Choice and Welfare, 12: $277-292$.

Shapley, L. S. and Scarf, H. (1974): "On Cores and Indivisibility." Journal of Mathematical Economics, 1: 23-28.

Sönmez, T. (1996): "Implementation in Generalized Matching Problems." Journal of Mathematical Economics, 26: 429-439.

Thomson, W. (1999): "Monotonic Extensions on Economic Domains." Review of Economic Design, 4: 13-33.

Yamato, T. (1992): "On Nash Implementation of Social Choice Correspondences." Games and Economic Behavior, 4: 484-492. 